\title{
Limitations of Flight Path Reconstruction techniques
}

\author{
C KAMALI $^{1, *(D)}$ and EROL OZGER ${ }^{2}$ \\ ${ }^{1}$ CSIR-National Aerospace Laboratory, Bangalore 560 017, India \\ ${ }^{2}$ Technische Hochschule Ingolstadt, Ingolstadt, Germany \\ e-mail: ckamali@nal.res.in; erol.oezger@thi.de
}

MS received 5 October 2017; revised 17 July 2018; accepted 11 October 2018; published online 22 January 2019

\begin{abstract}
The Flight Path Reconstruction (FPR) techniques are performed to verify the data compatibility check and post-flight. This is often achieved by calibrating the onboard sensors such as inertial and airdata sensors. In this paper, the limitations of FPR techniques in terms of Maximum Likelihood Estimation (MLE) and Extended Kalman Filter (EKF) and Unscented Kalman Filter (UKF) have been reported. To demonstrate the FPR and sensor calibration, kinematic trajectory simulations with wind box type maneuvers have been performed. It is also shown as how a kinematic simulation is valid for the studies carried out in this work.
\end{abstract}

Keywords. Flight path reconstruction; FPR; EKF; UKF; MLE; sensor calibration; wind box maneuvers.

\section{Introduction}

Modeling and simulation of dynamical systems make the design and analysis very cost effective. One of the essential areas of modeling and simulation is system identification/parameter estimation [1-4]. Although it applies to all physical systems, this work is centered around aircraft dynamical systems. The flight path reconstruction is primarily performed to calibrate the flight sensors $[1,5]$. The sensor measurements, in turn, are used to estimate the flight model parameters through which one can build a simulation model for the aircraft. If these sensors have systematic errors such as bias and scale factors, it results in poor estimation of model parameters required for building up the simulation model. Hence, FPR techniques have been employed to calibrate the sensors against systematic errors. The FPR can be performed using optimization-based techniques such as the MLE also called Output Error Method and Kalman Filter (KF) based methods.

The FPR using MLE method is discussed in [1], and it is claimed in [1] that the MLE performs better than KF methods as $\mathrm{KF}$ is an involved procedure and apriori knowledge on noise statistics is required for its implementation. But in [1], the wind estimation is not taken into consideration. Experiences on flight testing of a high-performance aircraft show that the FPR using MLE works well for low angles of attack and in the presence of steady winds that are not varying with altitude [6]. But to achieve high angles of attack, wind up turn maneuvers are performed where the wind significantly varies with altitude. It is observed in [6] that the MLE based method is not able to estimate varying winds with altitude and hence

*For correspondence results in poor FPR. Whereas the KF based techniques [6-9] have been shown to perform better under these conditions. In references [6-9], KF based techniques have been developed for air data calibration and accuracy determination of airdata system of a high-performance aircraft. But in [6-9], more accurate inertial sensors have been used to calibrate the airdata sensors. Although they have been shown to work on high angles of attack with varying winds, the limitation of the techniques have not yet been evaluated. Also, the scale factors in the angle of attack sensors, bias and scale factors in the angle of sideslip and bias and scale factors in True Air Speed (TAS) were not estimated in [6-9]. This is because adding more states to KF led to unobservable Angle of Attack (AoA) scale factor, AoSS bias and scale factors, TAS bias and scale factors, for some of the high-performance maneuvers.

Moreover, the work reported in [6-9] did not require the estimation of above parameters as the sensors are of good quality, it is of interest to include them too in the estimation to know the limitations of MLE and KF. Most papers discuss FPR and data compatibility using flight test data [10-14], but the limitations with respect to maneuvers are not widely discussed. Hence flight path reconstruction using MLE, EKF or UKF is not a nascent work [10-14], but their sensitivity to the type of maneuvers is.

A multi-sensor-based approach for the calibration of airdata systems has been reported in [14] using EKF and UKF. In [14], the conclusion states that the UKF performs better than EKF for airdata calibration. Also in [11-14], mostly flight data is used, and the true values of bias and scale factor parameters are not known. Moreover, the TAS bias and scale factors are not considered in the estimation. The wind estimation is again not dealt with [11, 12,14], and the maneuver types and limitations are not discussed [11-14]. Hence the 
unique contribution of this paper is that various FPR techniques such as MLE and EKF and UKF in a 24 state formulation are tested in a simulation environment to evaluate and compare their performance as well as to define their limitations when the truth is known. This is carried out systematically for six flyable maneuvers considering background wind estimation and all sensor errors including TAS bias and scale factors. The MLE implemented in this work uses the MATLAB code provided in [1] under chapter 4 and chapter 10 . The MLE code is extended for a 24 state estimation for this work. The codes for EKF and UKF are developed independently by the authors of this work.

It is for the first-time limitations of FPR techniques have been evaluated using a simulation framework using wind box maneuvers. The simulation is based on trajectory generation and kinematics so that one does not need a full 6 DOF simulation to prove FPR. The results have been verified with a high fidelity aircraft dynamics. Hence, the framework is very helpful to evaluate: which technique performs better under what framework or for what flight maneuvers.

The organization of the article is as follows.

Section 2 explains the kinematic simulation; section 3 explains modeling for filtering, section 4 discusses the results. Section 5 brings out the verification of findings using a six Degrees of Freedom (DoF) simulation. Section 6 highlights the findings and recommendations. In Appendix, Equations of Motions (EoM) and the required derivations are covered.

\section{Kinematic simulation}

To evaluate various flight path reconstruction approaches, a simulation environment is set-up where all essential parameters are under close control and the true parameters values to be estimated are known. For this, a trajectory simulation is build up since an aircraft simulation would require too much effort in flight path management which is not necessary for this work. Hence, in this work, a kinematic simulation environment is developed that may provide any maneuvers such as wind box type using trajectory generation. This aids the evaluation of FPR technique for any kind of flying vehicles such as a fixed wing, rotary wing, helicopter or any kind.

A trajectory is composed of simple segments such as a straight heading or a turn with or without climb angle as a function of time. The trajectory information of the aircraft position is thus given in geodetic coordinates $\mathrm{X}_{\text {Earth }}$.

$$
\vec{X}_{\text {Earth }}(t)=\left(\begin{array}{c}
x_{\text {North }}(t) \\
x_{\text {East }}(t) \\
x_{\text {Down }}(t)
\end{array}\right)
$$

Then this time dependant position information is differentiated two times to give rates and accelerations in geodetic coordinates

$$
\vec{X}_{\text {Earth }}(t) \stackrel{d / d t}{\longrightarrow} \dot{\vec{X}}_{\text {Earth }}(t) \stackrel{d / d t}{\longrightarrow} \stackrel{\vec{X}}{E a r t h}(t)
$$

Azimuth $\chi$ and climb angle $\gamma$ are calculated together with the total on ground speed $\mathrm{V}_{\text {Ground }}$.

$$
\begin{gathered}
V_{\text {Ground }}=\sqrt{\left(\dot{x}_{\text {North }}\right)^{2}+\left(\dot{x}_{\text {East }}\right)^{2}+\left(\dot{x}_{\text {Down }}\right)^{2}} \\
\gamma=\arcsin \left(\frac{-\dot{x}_{\text {Down }}}{V_{\text {Ground }}}\right) \\
\chi=\arctan \left(\frac{\dot{x}_{\text {East }}}{\dot{x}_{\text {North }}}\right)
\end{gathered}
$$

Aircraft orientation quantified by the Euler angles are introduced in a simple manner. The bank angle $\varphi$ depends on turning motion of the aircraft, namely

$$
\phi=\arccos \left(\frac{1}{N_{Z, \text { Aero }}}\right), \text { with } N_{Z, \text { Aero }}=\sqrt{1+\left(\frac{V_{\text {Ground }} \dot{\chi}}{g}\right)^{2}}
$$

The pitch angle $\theta$ depends on both the climb angle $\gamma$ and a compensating term to account for speed $\mathrm{V}_{\text {Ground }}$ and load factor during turning $\mathrm{N}_{\mathrm{Z} \text {,Aero }}$ with an appropriate constant $\mathrm{K}_{1}$ accouting for mass, density and aircraft lift gradient.

$$
\theta=\frac{K_{1} \cdot N_{Z, \text { Aero }}}{V_{\text {Ground }}^{2}}+\gamma
$$

The heading $\psi$ depends on the azimuth $\chi$ and its turn rate $\mathrm{d} \chi / \mathrm{dt}$ with a constant $\mathrm{K}_{2}$ to achieve approximately a coordinated turn with low angles of sideslip $\beta$.

$$
\psi=\chi+K_{2} \dot{\chi}
$$

Besides, dynamic maneuvering of the aircraft is emulated by the ability to superpose anytime doublets of varying amplitude and length on all three Euler angles. Up to now, all trajectory information is only in earth-fixed coordinated with Euler angle information. Now, the Euler angles are used together with a wind profile to compute the aircraft body fixed dynamic states.

The direction cosine matrix (DCM) $\mathrm{M}_{\text {Earth2AC }}$ is written in terms of the rotation matrix that describes the orientation of the body coordinates frame with respect to the inertial/navigation frame

$$
\begin{aligned}
M_{\text {Earth } 2 A C}= & \left(\begin{array}{ccc}
1 & 0 & 0 \\
0 & \cos \phi & \sin \phi \\
0 & -\sin \phi & \cos \phi
\end{array}\right) \\
& \cdot\left(\begin{array}{ccc}
\cos \theta & 0 & -\sin \theta \\
0 & 1 & 0 \\
\sin \theta & 0 & \cos \theta
\end{array}\right) \\
& \cdot\left(\begin{array}{ccc}
\cos \psi & \sin \psi & 0 \\
-\sin \psi & \cos \psi & 0 \\
0 & 0 & 1
\end{array}\right)
\end{aligned}
$$


Aircraft body accelerations as measured by the inertial measurement unit $\mathrm{N}_{\mathrm{X}}, \mathrm{N}_{\mathrm{y}}, \mathrm{N}_{\mathrm{z}}$ are calculated according to

$$
\left(\begin{array}{l}
N_{X} \\
N_{Y} \\
N_{Z}
\end{array}\right)=M_{\text {Earth2AC }} \cdot\left[\frac{1}{g} \ddot{\vec{X}}_{\text {Earth }}-\left(\begin{array}{l}
0 \\
0 \\
1
\end{array}\right)\right]
$$

Relative velocity as measured by aircraft sensors are determined together with the wind profile $\mathrm{W}=\left(\mathrm{W}_{\text {North }}\right.$, $\left.\mathrm{W}_{\text {East }}, \mathrm{W}_{\text {Down }}\right)^{\mathrm{T}}$

$$
\dot{\vec{X}}_{A C}=M_{\text {Earth } 2 A C}\left(\dot{\vec{X}}_{\text {Earth }}-\vec{W}\right)
$$

Airdata measurements $\left(\mathrm{V}_{\text {Aero }}, \alpha, \beta\right)$ can now be deduced accordingly,

$$
V_{\text {Aero }}=\left|\dot{\vec{X}}_{A C}\right|, \alpha=\arctan \left(\frac{\dot{z}_{A C}}{\dot{x}_{A C}}\right), \beta=\arcsin \left(\frac{\dot{y}_{A C}}{V_{\text {Aero }}}\right)
$$

Finally, aircraft angular rates (p,q,r) are computed by means of

$$
\begin{aligned}
\left(\begin{array}{c}
p \\
q \\
r
\end{array}\right) & =M_{\text {Euler }}^{T}\left(\begin{array}{c}
\dot{\phi} \\
\dot{\theta} \\
\dot{\psi}
\end{array}\right), \\
M_{\text {Euler }} & =\left(\begin{array}{ccc}
1 & \sin \phi \tan \theta & \cos \phi \tan \theta \\
0 & \cos \phi & -\sin \phi \\
0 & \sin \phi / \cos \theta & \cos \phi / \cos \theta
\end{array}\right)
\end{aligned}
$$

Furthermore, it is possible to impose any sort of error model on all parameters including noise to model a certain measurement behavior. As soon as the maneuver path in terms of the earth fixed positions and the Euler angles as a function of time are given the complete simulation data as described above can be determined and deduced.

\section{Modelling for filtering}

The filters for FPR together with calibration of inertial and airdata sensors essentially include the following states: Three axes position, Three axes velocity, Three axes attitude, Three axes wind, Three axes accelerometer bias, Three axes gyro bias, AoA bias, AoA scale factor, AoSS bias, AoSS scale factor, TAS bias and TAS scale factor.

\subsection{Filter model}

Kinematic equations of aircraft motion $[1,15]$ is the fundamental for FPR. The states of the filter in vector form are:

$$
x=\left[\begin{array}{llllll}
V & P & \Phi & I_{B} & A_{B} & W
\end{array}\right]
$$

where

$$
\begin{aligned}
& V=\left[\begin{array}{lll}
u & v & w
\end{array}\right] \\
& P=\left[\begin{array}{lll}
x_{p} & y_{p} & z_{p}
\end{array}\right] \\
& \Phi=\left[\begin{array}{lll}
\phi & \theta & \psi
\end{array}\right] \\
& I_{B}=\left[\begin{array}{llllll}
p_{b} & q_{b} & r_{b} & N_{x b} & N_{y b} & N_{z b}
\end{array}\right] \\
& A_{B}=\left[\begin{array}{llllll}
\alpha_{b} & \alpha_{s} & \beta_{b} & \beta_{s} & V T_{b} & V T_{s}
\end{array}\right] \\
& W=\left[\begin{array}{lll}
u_{w i} & v_{w i} & w_{w i}
\end{array}\right] \\
& \dot{V}=-\omega \times V+G+f_{b}+n 1
\end{aligned}
$$

where

$$
\begin{aligned}
& \omega=\operatorname{skew}\left(f_{g}\right) \\
& f_{g}=\left[\begin{array}{lll}
p-p_{b} & q-q_{b} & r-r_{b}
\end{array}\right] \\
& f_{b}=\left[\begin{array}{lll}
N_{x}-N_{x b} & N_{y}-N_{y b} & N_{z}-N_{z b}
\end{array}\right] \\
& G=M_{\text {Earth } 2 A C}\left[\begin{array}{l}
0 \\
0 \\
g
\end{array}\right] \\
& \dot{P}=V+n 1 \\
& \dot{\Phi}=M_{\text {Euler }}^{T} f_{g}^{T}+n 1 \\
& \dot{I}_{B}=0 \quad \dot{A}_{B}=0 \quad \dot{W}=0
\end{aligned}
$$

where $\mathrm{n} 1$ is Gaussian, zero mean process white noise.

The measurements for the filter in vector form are:

$$
A D S=\left(T A S, \alpha_{m}, \beta_{m}\right)
$$

where $V_{m}=$ measured inertial velocities, $P_{m}=$ measured position, $\Phi_{m}=$ measured attitudes.

Therefore, the measurement vector for the filters is as follows:

$$
Z=\left[\begin{array}{llll}
A D S & V_{m} & P_{m} & \Phi_{m}
\end{array}\right]
$$

The measurement equations for airdata can be understood from Eqs. (11) and (12). The air data velocities are calculated from the estimated body velocities and estimated winds using Eq. (11). In Eq. (12), the LHS represents the measured ADS, and the right hand of Eq. (12) comprise of airdata velocities estimated using Eq. (11) along with scale factors and bias. The other measurement equations are straight forward as the states are directly related to the measurements:

- Estimated body velocities can be equated to measured inertial velocities after transforming them to the body frame.

- Estimated positions can be equated to measured positions. 
- Estimated Euler angles can be equated to measured Euler angles

$$
\begin{aligned}
T A S & =|\stackrel{\overrightarrow{\dot{X}}}{A C}| \cdot \tilde{V}_{\text {Tscale }}+\tilde{V}_{\text {Tbias }}+n 2, \\
\alpha_{m} & =\arctan \left(\frac{\tilde{\dot{\vec{z}}}_{A C}}{\tilde{\dot{x}}_{A C}}\right) \cdot \tilde{\alpha}_{\text {scale }}+\tilde{\alpha}_{\text {bias }}+n 2, \\
\beta_{m} & =\arcsin \left(\frac{\tilde{\dot{y}}_{A C}}{\tilde{V}_{\text {Aero }}}\right) \cdot \tilde{\beta}_{\text {scale }}+\tilde{\beta}_{\text {bias }}+n 2 \\
V_{m} & =\tilde{M}_{\text {Earth } 2 A C}^{T} \tilde{V}+n 2 \\
P_{m} & =\tilde{P}+n 2 \\
\Phi_{m} & =\tilde{\Phi}+n 2
\end{aligned}
$$

where $\sim$ represents predicted states, $n 2$ is Gaussian, zero mean measurement white noise.

\subsection{Filter parameter tuning}

The process noise covariance for velocity dynamics depends on Gaussian noise and bias present in both accelerometer, gyro measurements. Similarly for the position dynamics. This covariance predominantly increases with either increasing values of accelerometer bias or with increasing variance of the white noise present in accelerometer measurements or both. The tuning of process noise covariance is carried out by typically squaring the values of standard deviation of noise present in accelerometer measurements and then increase the covariance values until filter convergence is reached. The process noise covariance for Euler angle dynamics depends on the noise and bias values present in gyro measurements. This covariance increase with either increasing values of gyro bias or with increasing variance of the white noise or both.

The following are the tuning parameters used for process noise covariance of EKF and UKF as provided in table 1. Please note that the process noise covariance for wind states, bias and scale factors are set to zero. One can also use a small value of the order of 1e-15. Nevertheless using zero value does not cause any mathematical divergence problems to the algorithm. The filter settings for EKF and UKF are identical.

The values of the measurement noise covariance for airdata measurements depends on the systematic errors and the Gaussian noise present in them. The higher the values of bias and scale factors/variance of the noise, the higher the measurement noise covariance. All inertial measure-

\begin{tabular}{|c|c|c|c|}
\hline State & Units for state & $\begin{array}{l}\text { Process } \\
\text { noise } \\
\text { covariance }\end{array}$ & Actual covariance \\
\hline $\mathrm{V}$ & $\mathrm{m} / \mathrm{s}$ & $1 e-4$ & $\begin{array}{l}\text { Here both gyro and } \\
\text { accelerometer noise } \\
\text { and winds act as } \\
\text { process noise: } \\
\text { The variance of white } \\
\text { noise added to } \\
\text { accelerometer: } \\
1.0000 \mathrm{e}-06 \\
\text { The variance of white } \\
\text { noise added to gyro: } \\
5.7300 \mathrm{e}-09\end{array}$ \\
\hline $\mathrm{W}$ & $\mathrm{m} / \mathrm{s}$ & 0 & 0 \\
\hline $\mathrm{I}_{\mathrm{B}}$ & $\begin{array}{c}\text { Degrees for gyro } \\
\text { biases, } \mathrm{m} / \mathrm{s}^{2} \text { for } \\
\text { accelerometer } \\
\text { biases }\end{array}$ & 0 & 0 \\
\hline $\mathrm{A}_{\mathrm{B}}$ & $\begin{array}{c}\mathrm{m} / \mathrm{s} \text { for TAS } \\
\text { degrees for } \\
\text { aoa,aoss bias }\end{array}$ & 0 & 0 \\
\hline$\Phi$ & Degrees & 0.00573 & $\begin{array}{c}\text { The variance of white } \\
\text { noise added to gyro: } \\
5.7300 \mathrm{e}-09\end{array}$ \\
\hline $\mathrm{P}$ & meters & $1 e-3$ & $\begin{array}{c}\text { Here again both gyro } \\
\text { and accelerometer } \\
\text { noise and winds act } \\
\text { as process noise: } \\
\text { The variance of white } \\
\text { noise added to } \\
\text { accelerometer: } \\
1.0000 \mathrm{e}-06 \\
\text { The variance of white } \\
\text { noise added to gyro: } \\
5.7300 \mathrm{e}-09\end{array}$ \\
\hline
\end{tabular}
ments have only white noise, and hence they can be believed, and their covariance values are very low. The following are the measurement noise covariance for EKF, UKF as in table 2 .
Table 1. Process noise covariance for the EKF/UKF.

The Filter error covariance matrix can be started with low values for those states whose initial values are available. This will enable lower transients during the initial stages of parameter convergence. For those states, whose initial values are not known, one can start with higher values of covariance. Higher covariance will enable faster convergence but will introduce higher transients during the initial stages of parameter convergence. If the initial transients for those parameters are too high, one can reduce the values of error covariance to strike a balance.

\subsection{Filter initial conditions}

The initial conditions play a significant role in the convergence of the filter. The initial conditions are set as zeros for all unknown biases. The initial conditions for the remaining states are available from sensor measurements. 
Table 2. Measurement noise covariance for the EKF/UKF.

\begin{tabular}{lccc}
\hline State & Units for state & Measurement noise covariance & Actual covariance of the measurement \\
\hline ADS & $\begin{array}{c}\text { meters/s for TAS } \\
\text { degrees for aoa, aoss } \\
\text { meters/s }\end{array}$ & $(0.010 .00570 .00057)$ & $(1.0000 \mathrm{e}-045.7300 \mathrm{e}-095.7300 \mathrm{e}-09)$ \\
$\mathrm{V}_{\mathrm{m}}$ & meters & $(1 \mathrm{e}-91 \mathrm{e}-91 \mathrm{e}-9)$ & $(1.0000 \mathrm{e}-041.0000 \mathrm{e}-041.0000 \mathrm{e}-04)$ \\
$\mathrm{P}_{\mathrm{m}}$ & Degrees & $(1 \mathrm{e}-71 \mathrm{e}-71 \mathrm{e}-7)$ & $(0.00250 .00250 .0025)$ \\
$\Phi$ & $(5.73 \mathrm{e}-85.73 \mathrm{e}-85.73 \mathrm{e}-8)$ & $(5.7300 \mathrm{e}-055.7300 \mathrm{e}-055.7300 \mathrm{e}-05)$ \\
\hline
\end{tabular}

The following are the initial conditions used for the filters (EKF, UKF, and MLE) as in table 3.

\section{Results and discussion}

\subsection{Experimental set-up}

The aim of this work is to evaluate the limitations of FPR using Output Error Method and Filter Error Methods in a controlled environment where the truth is known for comparisons. The following are the maneuvers performed:

(1) Wings level maneuver

(2) Wings level maneuver with Euler doublets

(3) Wings level maneuver with Euler doublets, accelerations and decelerations

(4) Wind box maneuver

(5) Wind box maneuver with Euler doublets

(6) Wind box maneuver with Euler doublets, accelerations and decelerations

The first three maneuvers are based on a straight level segment. Maneuver 1 assumes constant pitch and roll angles with a constant speed of $50 \mathrm{~m} / \mathrm{s}$ with a duration of $20 \mathrm{~s}$. Maneuver 2 is like maneuver one but with additional doublets on the roll, pitch and yaw angles (e.g., roll angle amplitude $15^{\circ}$ with $3 \mathrm{~s}$ length, pitch angle amplitude $5^{\circ}$ with $4 \mathrm{~s}$ length and yaw angle amplitude $10^{\circ}$ with $5 \mathrm{~s}$ length). Maneuver 3 comprises all properties of maneuver 2 with an additional acceleration (from $50 \mathrm{~m} / \mathrm{s}$ to $80 \mathrm{~m} / \mathrm{s}$ in $20 \mathrm{~s}$ ) and a deceleration back to the initial at the same time. Therefore maneuver 3 has a duration of $40 \mathrm{~m} / \mathrm{s}$.

The fourth to sixth maneuvers are build up in the same philosophy as the first three ones with the difference that

Table 3. Initial Conditions used for the filter.

\begin{tabular}{lc}
\hline State & Initial condition \\
\hline $\mathrm{V}$ & Calculated from measured GPS velocities at time zero \\
$\mathrm{P}$ & Calculated from GPS position at time zero \\
$\Phi$ & Taken from measured Euler angles at time zero \\
$\mathrm{W}$ & 0 \\
$\mathrm{I}_{\mathrm{B}}$ & 0 \\
$\mathrm{~A}_{\mathrm{B}}$ & 0 \\
\hline
\end{tabular}

there is not one straight segment but four perpendicular segments connected by coordinated turns with $300 \mathrm{~m}$ turn radius with a roll angle of around $40^{\circ}$ during the turn. These four segments and their turn parts make up a box type maneuver path. Maneuver 4 is the wind box maneuver with wings level, and constant speed of $50 \mathrm{~m} / \mathrm{s}$ during straight segments and a roll angle build up during the turn with more than $80 \mathrm{~s}$ duration. Maneuver $5 \mathrm{com}$ prises additionally doublets of Euler angles (roll, pitch, and yaw angle as in maneuver 2) on all four straight segments and maneuver 6 includes all aspects of maneuver 5 with an additional acceleration (from $50 \mathrm{~m} / \mathrm{s}$ to $80 \mathrm{~m} / \mathrm{s}$ in $20 \mathrm{~s}$ ) and deceleration (from $80 \mathrm{~m} / \mathrm{s}$ to $50 \mathrm{~m} / \mathrm{s}$ in $20 \mathrm{~s}$ ) on each straight segment. Each segment takes around $40 \mathrm{~s}$ making up more than $160 \mathrm{~s}$ for the whole maneuver due to the turns.

All the 6 six maneuvers are performed at a constant altitude with constant north and east winds. Constant horizontal wind is indeed often assumed for flight path reconstruction because experience shows that in many cases the maneuver used are short enough to make this assumption valid. The constant altitude assumption also aids no variation in the wind. The sensor characteristics and steady winds used for simulation are as in table 4: These are the unknown parameters to be estimated for obtaining the correct flight path.

\subsection{Discussion of results}

This section presents results with respect to maneuver types $1-6$. The first three maneuvers $1-3$ are simple ones and the time history of aircraft states are not presented for them while figure 1 presents how the wind box maneuver looks like in the $\mathrm{X}-\mathrm{Y}$ plane. Figure 2 shows the time histories for a typical wind up turn maneuver with Euler doublets, accelerations and decelerations. Figure 3 shows estimation by UKF as an example, just to show the most important parameter variations during maneuvering, Red-True, GreenEstimated, Blue-Measured (containing sensor errors).

\section{Maneuver 1: Wings level}

The Equations of Motion and the necessary derivations for the kinematic simulation are presented (see Appendix). In a wings level maneuver, there are no rotations, and the altitude is maintained constant. 
Table 4. Sensor errors and winds used for simulation.

\begin{tabular}{lccc}
\hline Parameter & Bias & Scale factor & Standard deviation of the white noise \\
\hline Roll rate & $3 \mathrm{deg} / \mathrm{s}$ & - & $5.7300 \mathrm{e}-04 \mathrm{deg} / \mathrm{s}$ \\
Pitch rate & $-2 \mathrm{deg} / \mathrm{s}$ & - & $5.7300 \mathrm{e}-04 \mathrm{deg} / \mathrm{s}$ \\
Yaw rate & $3 \mathrm{deg} / \mathrm{s}$ & - & $5.7300 \mathrm{e}-04 \mathrm{deg} / \mathrm{s}$ \\
Forward acceleration & $-0.9 \mathrm{~m} / \mathrm{s}$ & - & $0.001 \mathrm{~m} / \mathrm{s}$ \\
Lateral acceleration & $0.7 \mathrm{~m} / \mathrm{s}$ & - & $0.001 \mathrm{~m} / \mathrm{s}$ \\
Normal acceleration & $-1.5 \mathrm{~m} / \mathrm{s}$ & - & $0.001 \mathrm{~m} / \mathrm{s}$ \\
AOA & $4.1 \mathrm{deg}$ & 1.1 & $5.7300 \mathrm{e}-04 \mathrm{deg}$ \\
AOSS & $5.9 \mathrm{deg}$ & 1.15 & $5.7300 \mathrm{e}-04 \mathrm{deg}$ \\
TAS & $10 \mathrm{~m} / \mathrm{s}$ & -05 & $0.01 \mathrm{~m} / \mathrm{s}$ \\
North Wind & $2.5 \mathrm{~m} / \mathrm{s}$ & - & - \\
East Wind & $2.5 \mathrm{~m} / \mathrm{s}$ & - & - \\
Down Wind & $0 \mathrm{~m} / \mathrm{s}$ & - & - \\
Bank angle & - & - & $0.0573 \mathrm{deg} / \mathrm{s}$ \\
Pitch angle & - & - & $0.0573 \mathrm{deg} / \mathrm{s}$ \\
Yaw angle & - & - & $0.0573 \mathrm{deg} / \mathrm{s}$ \\
Inertial velocity, X, Y, Z & - & $0.01 / \mathrm{s}$ \\
\hline
\end{tabular}

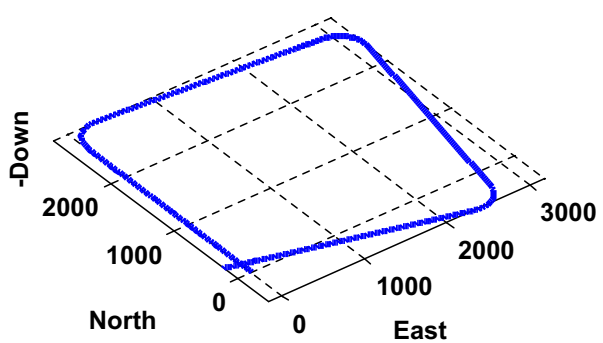

Figure 1. Wind box maneuver.

$$
\begin{aligned}
& V=\text { Const }, \alpha=\text { Const }, \beta=\text { Const } \\
& p=q=r=0
\end{aligned}
$$

Where Const means constant.

This maneuver is of $20 \mathrm{~s}$ duration. As can be seen in figures (4-6), that none of the techniques were able to estimate all the parameters perfectly. In bar charts 4-6 org means original values, man 1 ...man 6 means maneuver 1-6. This is mainly because the maneuver did not provide sufficient information in the data. The MLE can estimate the inertial sensors biases very well compared to EKF and UKF, even for a wings level flight. But MLE shows a higher correlation between some of the states. The MLE results are not compared in figures 4-5 as the results are completely diverging for airdata. Nevertheless, in figure 7 , inertial biases are compared with true values for MLE estimates.

\section{Maneuver 2: Wings level with Euler doublets}

This maneuver starts as wings level and in between some rotations in terms of Euler doublets (roll, pitch, yaw) are included in the maneuver to introduce rotation information in the data. Velocity and altitude remain constant. This is also 20-s duration. Here, As seen in bar charts (4-6) the EKF and UKF showed improvements in AoA bias, scale factor, AoSS scale factor and improved accuracy in inertial sensor biases. The MLE estimated the inertial sensor biases very well as in figure 7. But all airdata parameters were diverging in MLE. Hence none of the techniques were able to determine the winds and TAS bias and scale factor.

\section{Maneuver 3: Wings level with Euler doublets and accelerations, decelerations}

This also starts as wings level maneuver, where in addition to the rotations in terms of Euler doublets (roll, pitch, yaw), accelerations and decelerations are introduced to gain some more information in terms of speed. This maneuver is for a duration of $40 \mathrm{~s}$. Again, MLE couldn't bring any improvements in the air data parameters. The EKF and UKF started improving concerning airdata parameters but still, $\mathrm{u}$ wind can not be estimated by EKF and UKF as can be seen in bar charts (4-6). TAS bias is also not fully converged. The MLE estimated the inertial sensor biases very well as in figure 7 . Ideally MLE should have worked well on airdata parameters as well for this maneuver. So the reason why it failed is investigated. The states were reduced one by one, starting from TAS scale factor and then TAS bias. When the bias and scale factors in TAS were removed from the data and in the estimation process, MLE reported good results with respect to the rest of the unknown parameters. The experiment is repeated for maneuvers 1 and two as well. The results are plotted in the bar chart in figure 8 for maneuvers $1-3$. It is seen in figure 8 that MLE worked very well for maneuver3 without TAS bias and scale factors. When TAS bias and scale factors were introduced, the technique simply failed due to high correlations among parameters. 

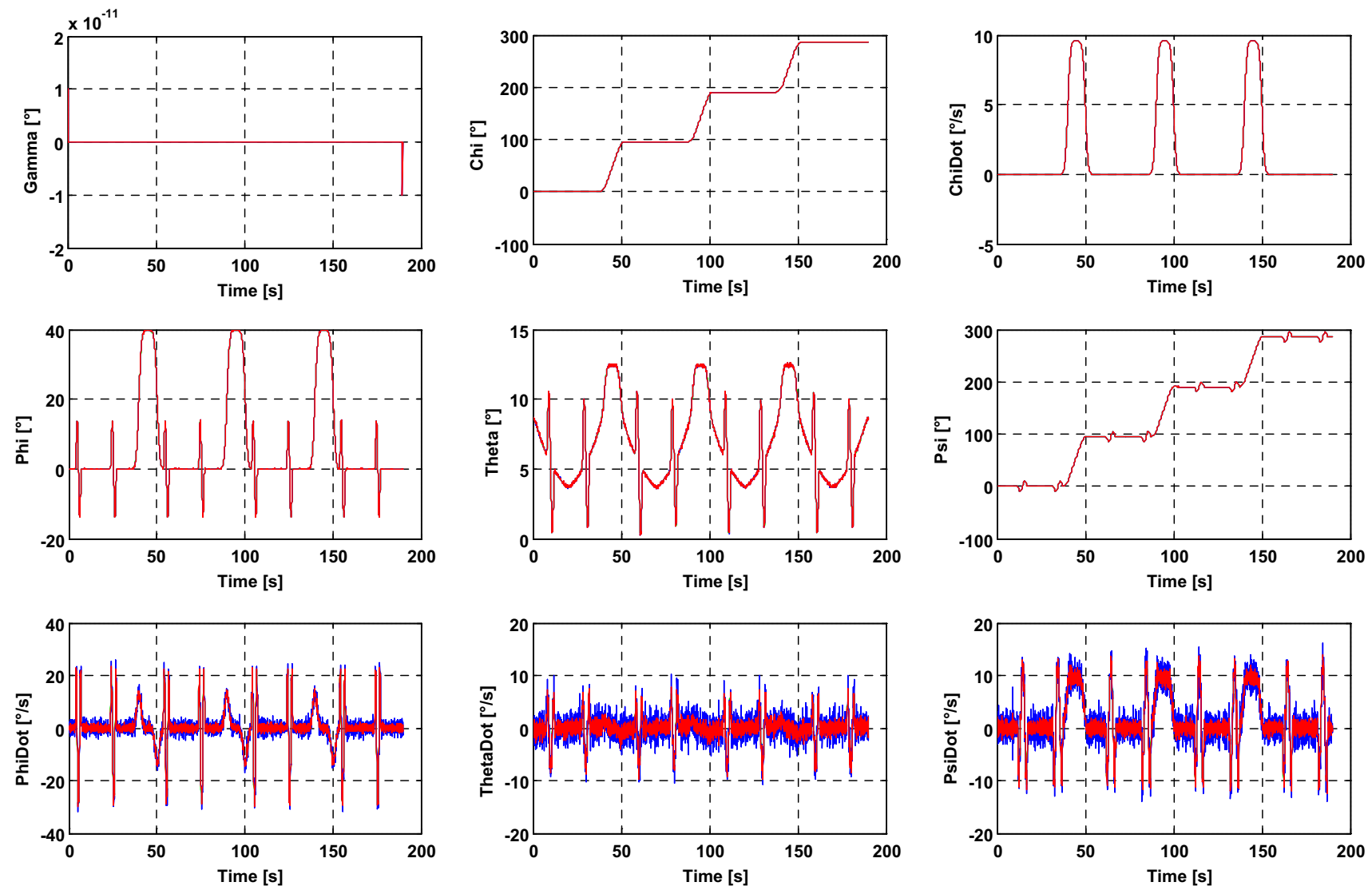

Figure 2. Time histories for a typical wind up turn maneuver with Euler doublets, accelerations and decelerations.
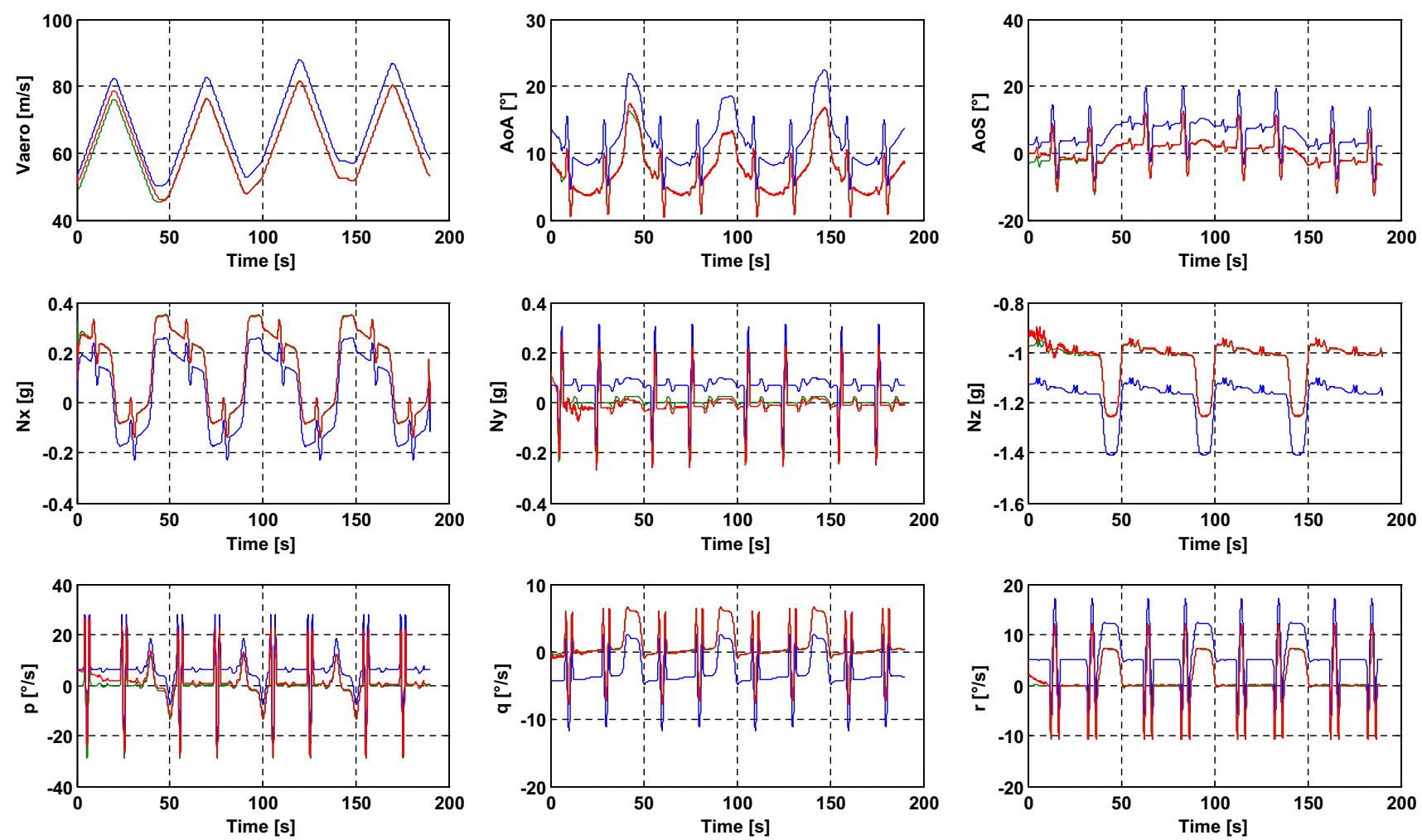

Figure 3. Estimation by UKF (example, just to show the most important parameter variations during maneuvering), Red-True, GreenEstimated, Blue-Measured (containing sensor errors). 

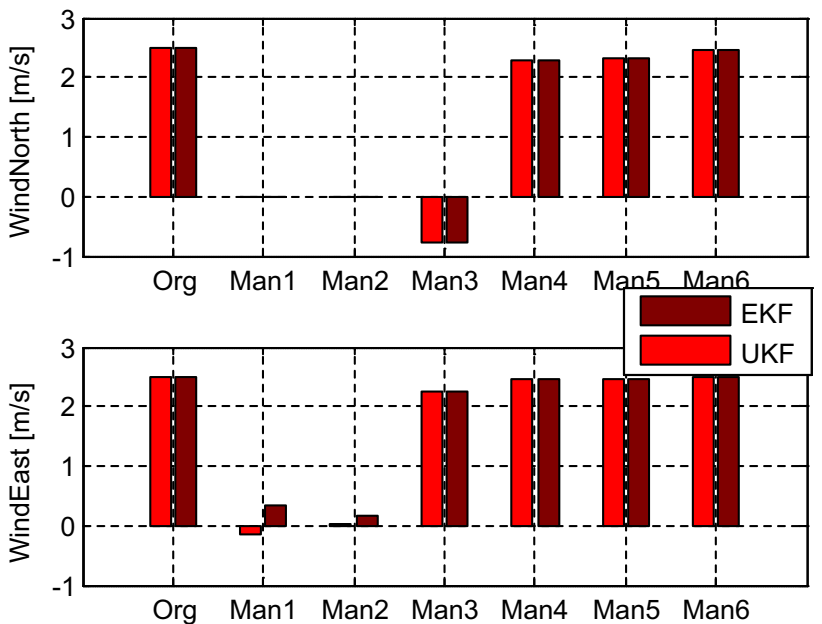

Figure 4. Wind estimation (Heading change necessary to estimate wind speed. Accel \& Decel or Euler doublets not necessary).

\section{Maneuver 4: Wind box}

This is of maneuver as shown in figure 1. There are four heading changes here. The translational velocities and rotational velocities change whenever there is a heading change. Otherwise, they remain constant. This is of duration 110 s. The MLE can not handle this big data. But still, for comparison sake, all three techniques are run for $110 \mathrm{~s}$. It is noted that the MLE started diverging with respect to all parameters. The reasons for divergence are longer data and inclusion of TAS bias and scale factor estimation. Hence, only $20 \mathrm{~s}$ data from wind box maneuver was fed to check the performance of MLE. This time, the inertial parameters were converging, and few of the airdata parameters such as beta bias, scale factors were converging. But winds and other airdata parameters are not estimable as the technique was not able to handle the correlation between states as discussed in case 3. Whereas the EKF and UKF results are improved with respect to all the estimated parameters including the north wind and velocity bias which was found to be bad for maneuvers 1-3. Since MLE is not suitable for handling wind box maneuvers which are of larger data length, it is dropped in the comparison in figures 2-3.

\section{Maneuver 5: Wind box with Euler doublets}

This is similar to Maneuver 4 but includes Euler doublets (roll, pitch, yaw) to get improved rotation information. The aim is to improve the inertial sensor biases estimated by EKF and UKF. This is of duration $110 \mathrm{~s}$. As can be seen from bar charts 4-6, the airdata parameters accuracy by EKF and UKF improved compared to maneuver four but the inertial parameters accuracy remained same as in
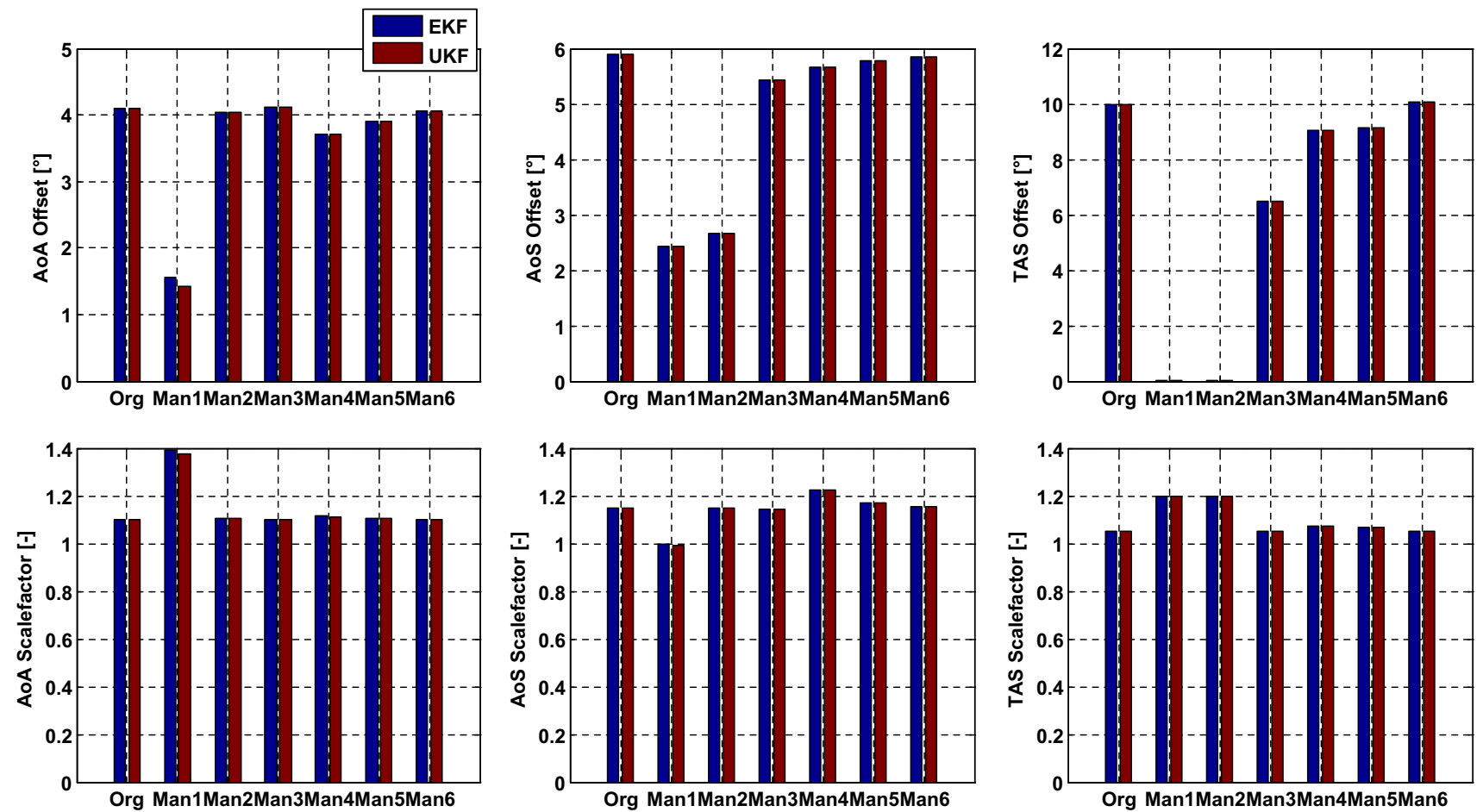

Figure 5. Airdata biases and scale factors estimation. (Wind estimation influences AoA, AoS and V offsets. Poorer prediction performance if no heading changes present. Accel \& Decel and Euler doublets improve slightly offset estimation. Scale estimation nearly independent of maneuver type.) 

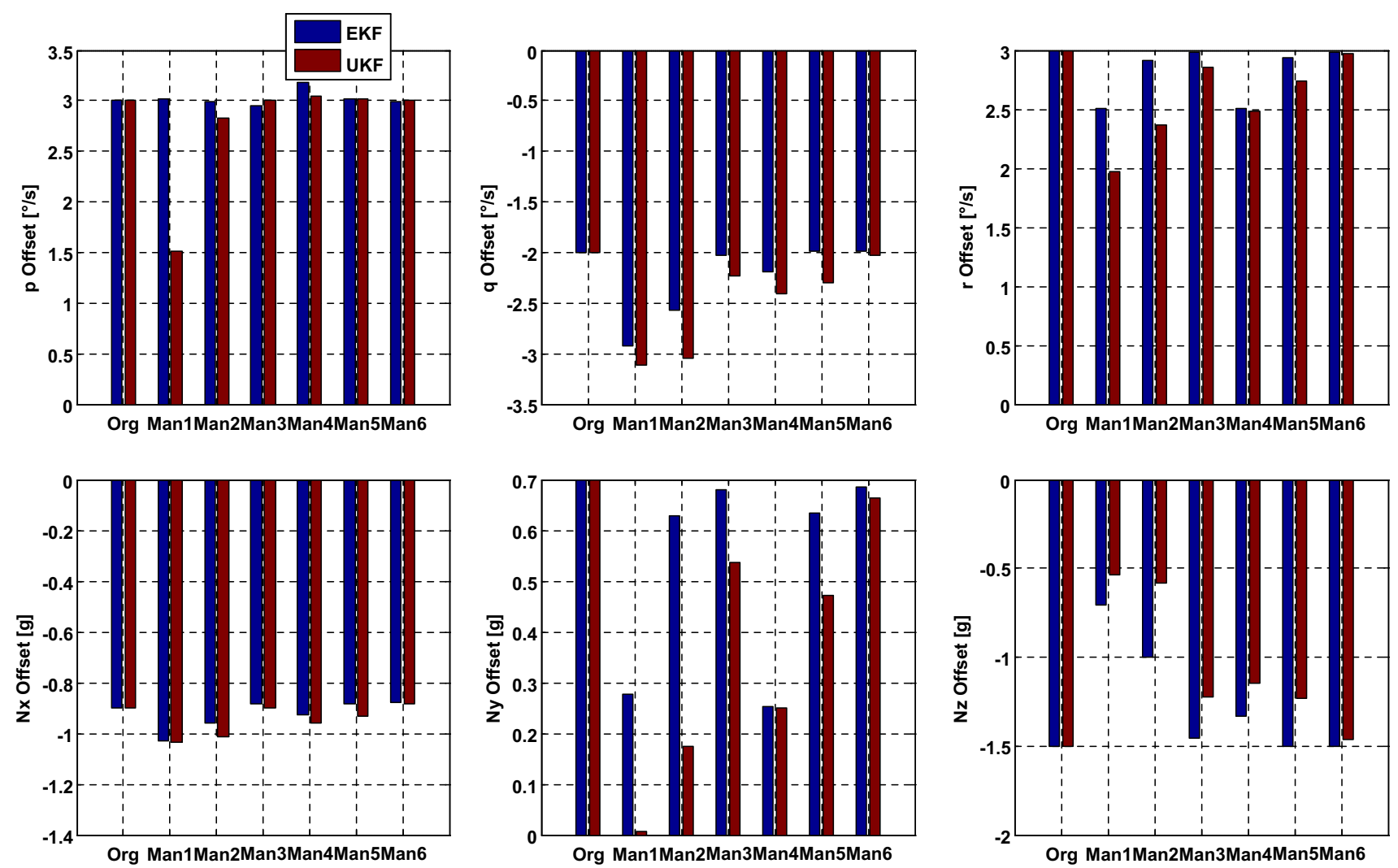

Figure 6. Inertial sensor bias estimation (Euler doublets and accel \& Decels improve estimation quality of IMU offset estimation, compare Man1 to 3 and Man 4 to 6).

maneuver 4. MLE is again completely divergent, and the discussions about MLE in maneuver 4 holds good for this maneuver also.

\section{Maneuver 6: Wind box with Euler doublets and acceler- ations and decelerations}

This is similar to Maneuver 5 but includes acceleration and decelerations to get speed information. It is noted that the Inertial sensor bias values have not improved much but the airdata parameters are estimated accurately by EKF and UKF as seen in figures 1-3. It is observed, that the inertial sensor biases converged very well between 20 and $40 \mathrm{~s}$. But beyond $40 \mathrm{~s}$ heading changes were performed for wind box maneuver. During that period, the inertial sensor biases slightly diverge from the converged values. As an example, figure 3 shows the most important parameter variations during maneuvering. The overall accuracy is excellent. Nevertheless, for reliable estimation of inertial parameters, it is desirable to perform wings level maneuver. Freeze those inertial bias values before performing wind box maneuver for airdata calibration. This leads to the best estimation of all the parameters by EKF or UKF. MLE is completely divergent for the obvious reasons as discussed in maneuvers 4,5 .

\section{Verification of findings using a six Degrees of Freedom (DoF) simulation}

In the current work, the conclusions are drawn from a kinematic simulation platform. This is because for a developmental aircraft one needs to have full aerodynamic data (wind tunnel/CFD) to make a six DoF simulation. This is often not so easy in places like academic institutions. Hence a kinematic simulation platform is developed in this work to design and develop FPR type algorithms. However, it is worthwhile to justify the kinematic simulation against a six DoF aircraft simulation so that the following doubts are cleared:

- Whether the flight path (horizontal) changes from the one that is shown in figure 1 when we perform maneuvers in a wind box maneuver.

- Whether plain windbox with default acceleration decelerations and attitude changes be enough to estimate all the parameters or still Euler doublets and accelerations and decelerations are required?

Hence the following maneuvers were performed in the six DoF simulation based on the preceding discussions.
V.1 Windbox
V.2 Windbox with Euler doublets 

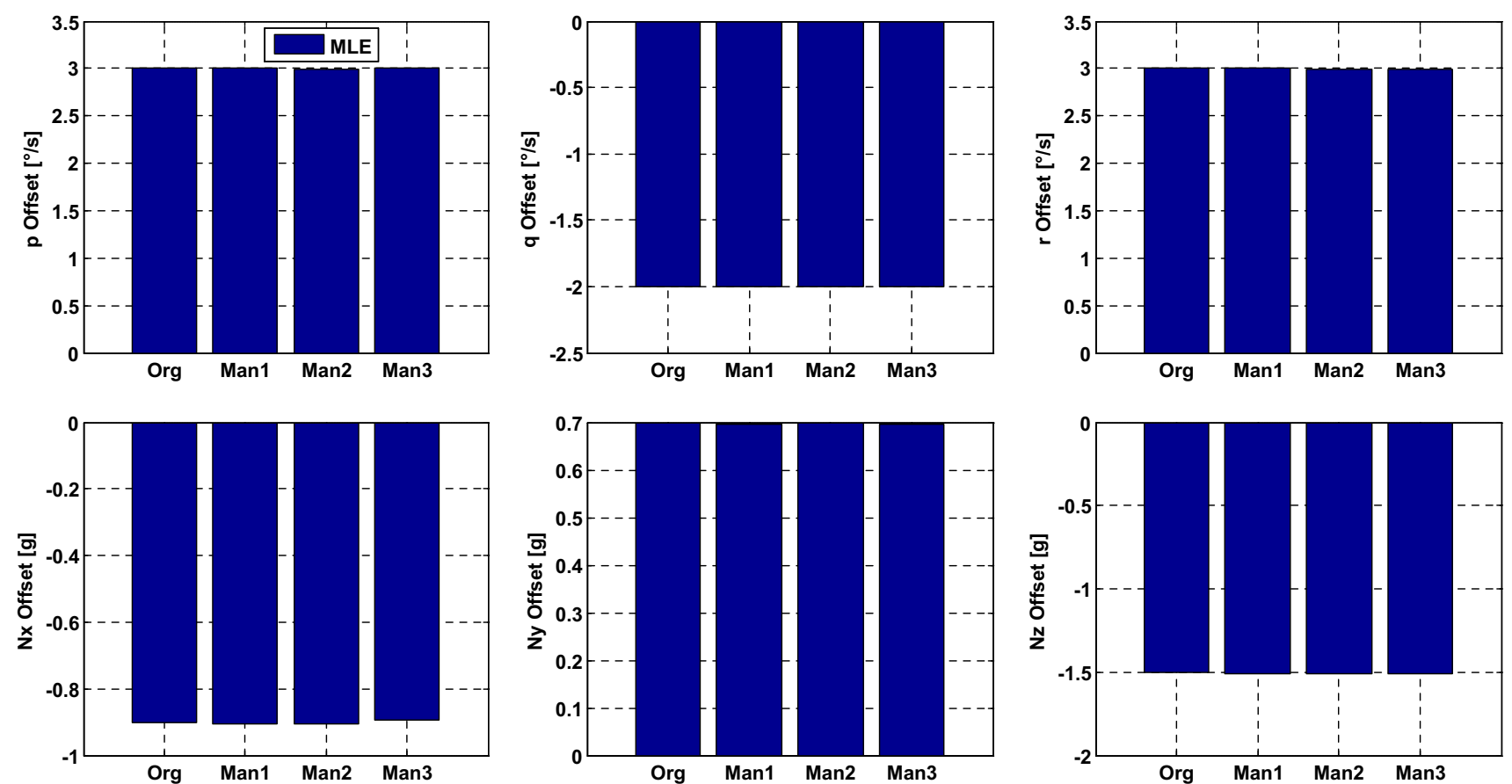

Figure 7. Inertial sensor bias estimation by MLE (Maneuver 1-3, proves that MLE is very good for inertial bias estimation with wings level maneuvers of shorter data length).
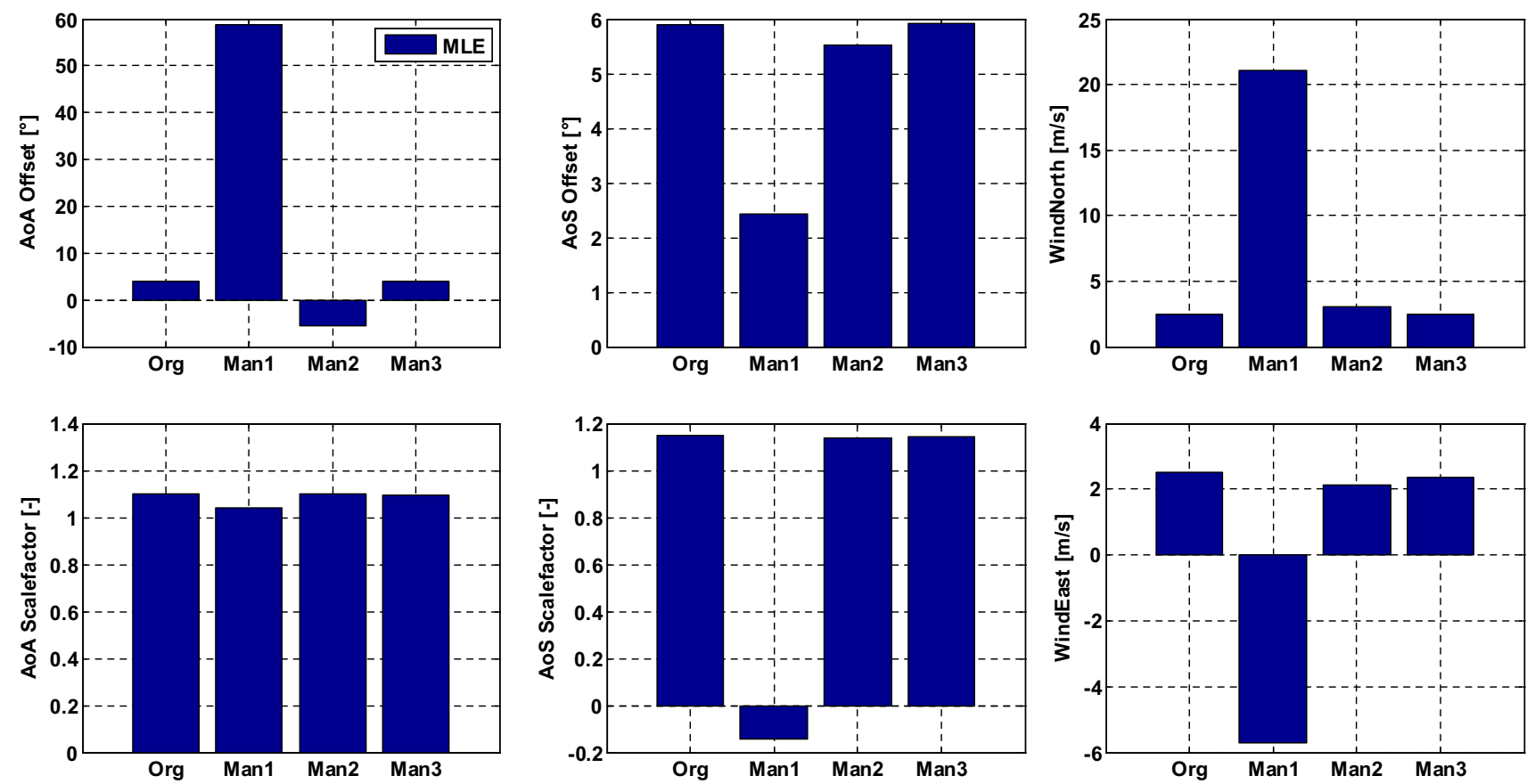

Figure 8. Flow angles bias and scale factor and wind estimation by MLE (Maneuver 1-3, Euler doublets and acceleration and decelerations are essential in estimating flow angle scale and bias and also winds).

The simulations are performed in the Engineer's in the Loop Simulation (ELS) facility for a high-performance fighter aircraft. Both V.1 and V.2 had sufficient accelerations and decelerations, and hence they were not additionally introduced unlike the case of kinematic simulation.
It is confirmed that from figure 9 that there is no change in the pattern of the wind box maneuver (due to the presence or absence of Euler doublets) in the horizontal plane for the maneuvers V.1 and V.2. Hence the assumptions made in the kinematic simulation is perfectly alright and is valid. 

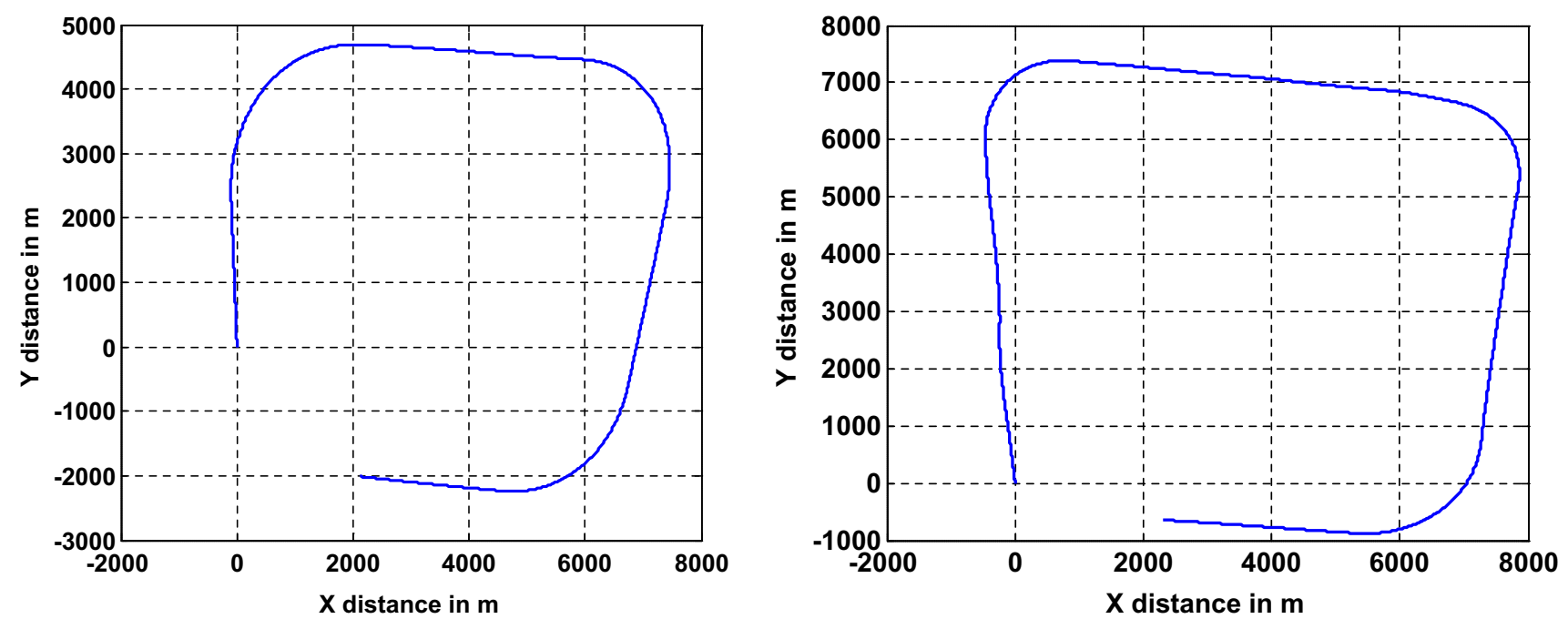

Figure 9. Manuvers V.1 and V.2 performed in ELS.

The north wind of magnitude $15 \mathrm{~m} / \mathrm{s}$ was injected in the simulation. When the pilot performed the maneuver, due to the presence of wind, there were default acceleration, decelerations, and attitude changes. It is confirmed from figure 10 that both maneuvers could estimate the winds as in the case of kinematic simulation.

Figure 11 shows the comparison of airdata bias and scale factor estimation against true values. It is seen that the Euler doublets improve offset estimation and scale is not much dependent on the maneuver type. This is again the same conclusion as that of kinematic simulation.

Figure 12 shows the inertial sensor bias in ELS since both maneuvers have accelerations, decelerations and attitude changes both of them estimated the inertial sensor biases accurately as in the case of kinematic simulation.
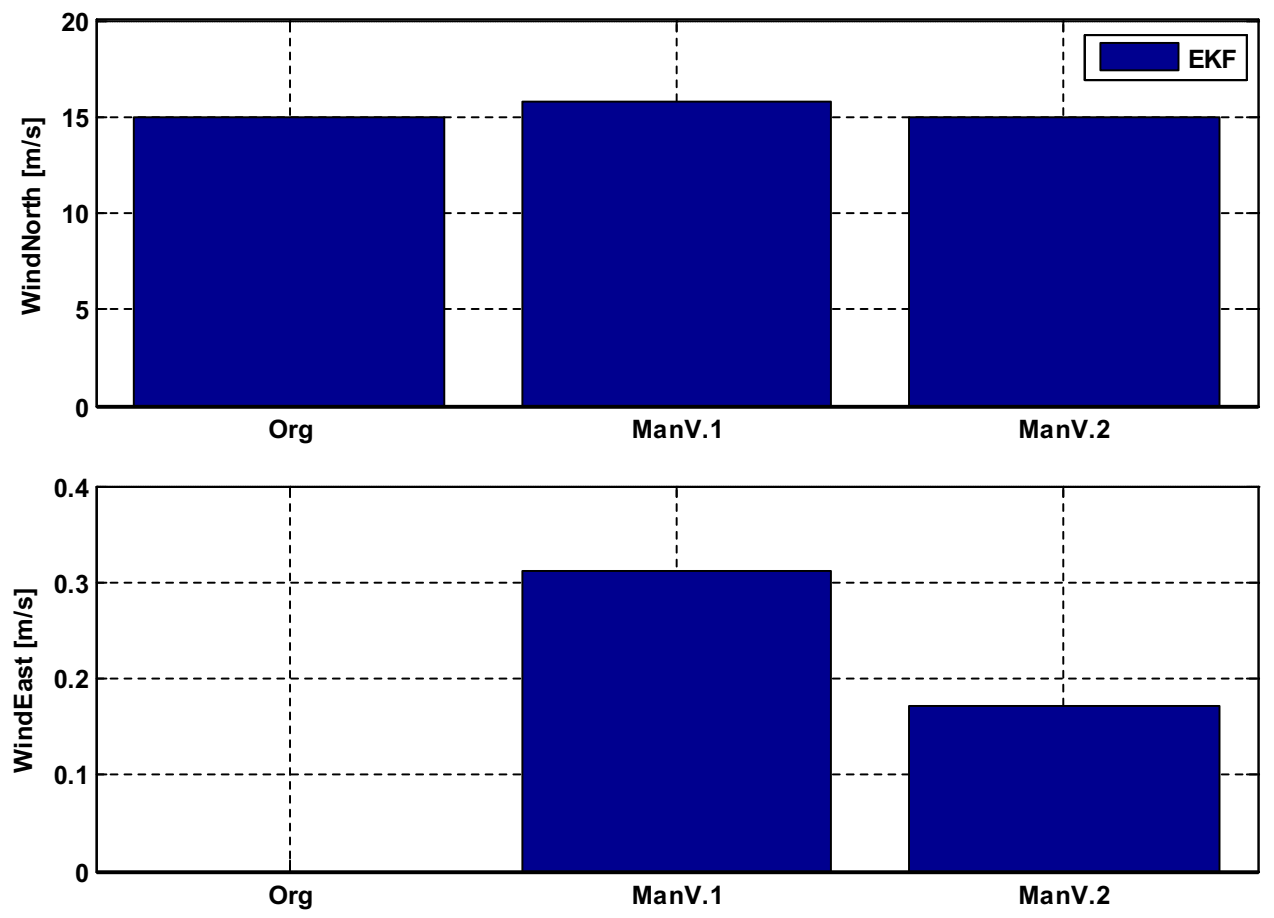

Figure 10. Wind estimation in ELS (Heading change necessary to estimate wind speed Accel\&decel or Euler doublets not necessary). 

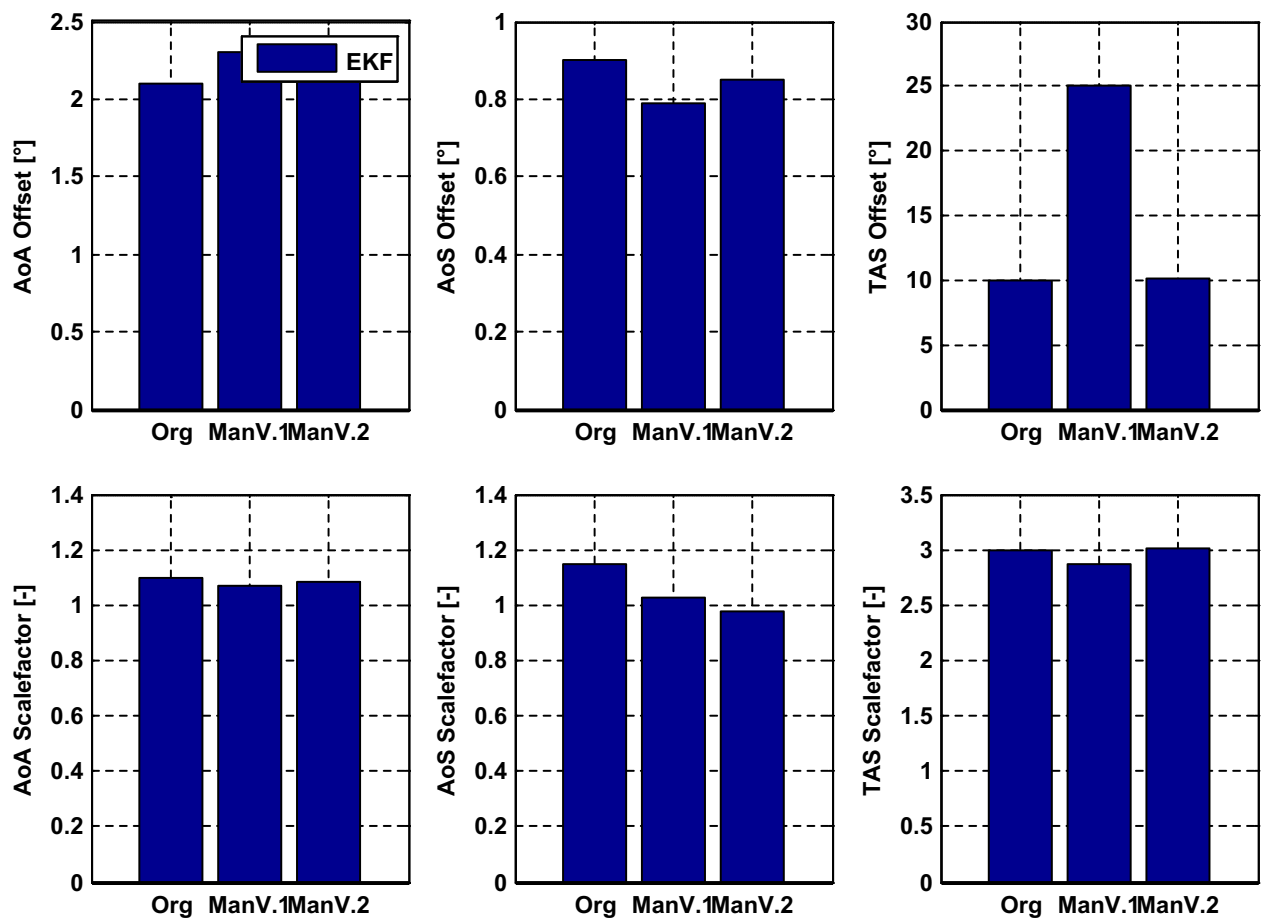

Figure 11. Airdata bias and scale estimation in ELS (Euler doublets improve offset estimation and Scale is not much dependent on the maneuver type).

Figure 13 shows the real-time convergence of all the states.

Hence the conclusions drawn from the kinematic simulations are found to be valid, and such a platform is more than sufficient to develop FPR algorithms in contrast to a high fidelity aircraft dynamics. Indeed this is also one of the significant findings of this work.
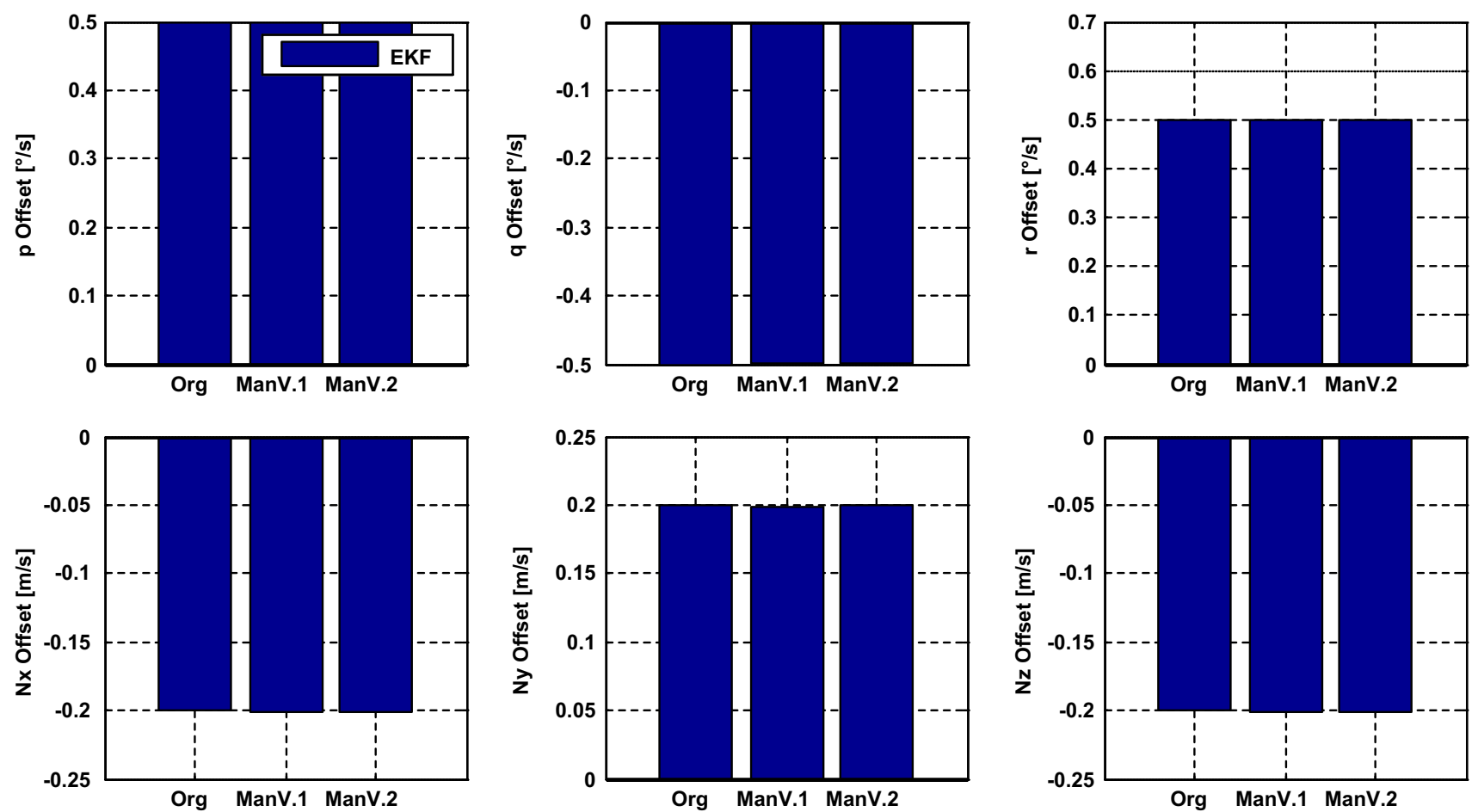

Figure 12. Inertial sensor bias estimation in ELS. 

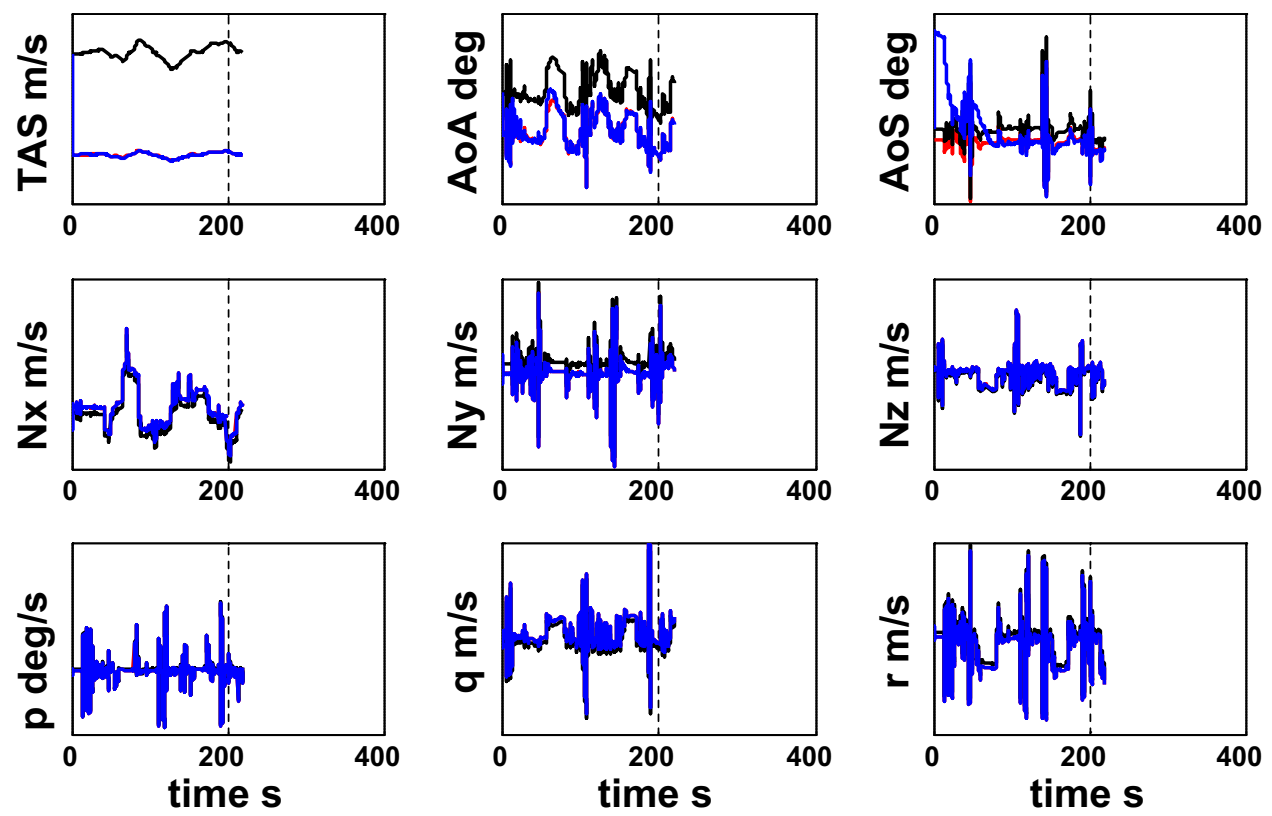

Figure 13. Estimation by EKF (example, just to show the most important parameter variations during maneuvering V.2), Red - True, Blue - Estimated, Black - Measured (Containing sensor errors).

\section{Conclusion}

The work investigated the limitations of 24 state FPR using MLE, EKF, and UKF using trajectory generation followed by kinematic simulation. The evaluation brought out the limitations of the techniques concerning the number of parameters they can estimate and the type of maneuvers required for a better estimation of all the unknown parameters. The work investigated FPR evaluation for worst sensor errors whose actual values are known. The following are the findings.

\subsection{Limitations of 24 state MLE}

- It is well known that Kalman filter was obtained by deriving the equations of the Maximum Likelihood Estimator for a very specific set of assumptions (linear model, Gaussian process, and measurement noise) but the MLE could not achieve the same performance when the TAS bias and scale factors were introduced.

- Could not handle data correlations.

- Could not handle longer data length such as in the wind box maneuver.

\subsection{Limitations of 24 state $E K F / U K F$}

- The wings level maneuvers could not estimate all the air data parameters.
- Does not work for shorter data length as they are recursive techniques and require some time for final convergence.

- Heading change makes wind estimation possible

- Euler doublets with accel \& decels improve inertial sensor bias estimation.

- Accel \& Decel improves TAS estimation (good wind estimation is a prerequisite).

- The wind box maneuvers made the inertial sensor biases which are already converged to slightly diverge once the heading changes are initiated.

- Filters need to be tuned, unlike MLE.

- Shows only rank 18 , nevertheless with maneuver types (6th maneuver) the observability is seen to increase practically.

\subsection{Recommendations}

- The simulation framework and the estimation algorithms developed here can be used for any type of flying vehicle as the results are validated using a high fidelity aircraft dynamics simulation.

- Comparing the six maneuvers, EKF and UKF both achieve the best performance for maneuver 6 , and the performance is almost similar. It is noted that the UKF performs identically to EKF as the nonlinearity involved in the maneuvers discussed for the FPR are not very high.

- The work was subsequently tested successfully using flight test data of a high-performance aircraft by simulating low-cost sensor characteristics. 


\section{Appendix}

The Equations of Motion (EOM) for the simulation is derived from point mass model as follows:

$$
\begin{gathered}
\dot{V} m=T \cos \alpha-D-W \sin \gamma \\
\dot{\gamma} m V=(L+T \sin \alpha) \cos \mu-W \cos \gamma \\
\dot{\chi} m V \cos \gamma=(L+T \sin \alpha) \sin \mu \\
\dot{x}=V \cos \gamma \cos \chi \\
\dot{y}=V \cos \gamma \sin \chi \\
\dot{h}=V \sin \gamma
\end{gathered}
$$

For constant flight path angle and small angles of attack Eq. (26) reduces to

$$
L \cos \mu=W \cos \gamma=m g \cos \gamma
$$

We know

$$
\begin{gathered}
N_{z}=\frac{L}{m g} \\
\sqrt{L^{2} \sin ^{2} \mu+L^{2} \cos \mu}=\sqrt{L^{2}}=L \\
\frac{V \dot{\chi}}{g}=\frac{L \sin \mu}{m g \cos \gamma}
\end{gathered}
$$

Substitute Eqs. (29) and (32) in Eq. (31)

$$
L=\sqrt{\left(\frac{V^{2} \dot{\chi}^{2}}{g^{2}}\right) m^{2} g^{2} \cos ^{2} \gamma+m^{2} g^{2} \cos ^{2} \gamma}
$$

Substitute (33) in (30)

$$
N_{Z}=\sqrt{\frac{\left(\frac{V^{2} \dot{\chi}^{2}}{g^{2}}\right) m^{2} g^{2} \cos ^{2} \gamma}{m^{2} g^{2}}+\frac{m^{2} g^{2} \cos ^{2} \gamma}{m^{2} g^{2}}}
$$

Hence

$$
\begin{aligned}
& N_{Z}=\sqrt{\left(\frac{V^{2} \dot{\chi}^{2}}{g^{2}}\right) \cos ^{2} \gamma+\cos ^{2} \gamma} \\
& N_{Z}=\sqrt{1+\left(\frac{V^{2} \dot{\chi}^{2}}{g^{2}}\right) \cos \gamma}
\end{aligned}
$$

Equation (30) can be written as:

$$
\begin{aligned}
N_{Z} & =\frac{0.5 \rho V^{2} C_{L \alpha} \alpha}{m g} \\
\theta & \approx \alpha=\frac{N_{Z} K}{V^{2}}+\gamma
\end{aligned}
$$

\section{Nomenclature}

$\begin{array}{ll}\vec{X}_{\text {Earth }} & \text { Position in the Earth coordinate } \\ \dot{\vec{X}}_{\text {Earth }}, V & \text { Velocity in the Earth coordinate } \\ \overrightarrow{\vec{X}}_{\text {Earth }} & \text { Accelerations in the Earth } \\ \Phi & \text { coordinate } \\ \phi & \text { Euler angles } \\ \theta & \text { Bank angle } \\ \psi & \text { Pitch angle } \\ \vec{g} & \text { Yaw angle } \\ \dot{\vec{X}} & \text { Gravity vector } \\ W & \text { Velocity in the body frame relative } \\ \alpha & \text { to wind } \\ \beta & \text { Wind vector in the inertial frame } \\ T A S & \text { Angle of attack } \\ V_{\text {Tbias }}, V_{T s c a l e} & \text { Sideslip angle } \\ \alpha_{b i a s} \alpha_{s c a l e} \beta_{b i a s} \beta_{\text {scale }} & \text { True Air Speed } \\ & \text { TAS bias/offset and scale factor } \\ u, v, w & \text { AoSS bias/offset, AoSS scale } \\ u_{w i}, v_{w i}, w_{w i} & \text { factor } \\ \mathrm{P} & \text { Velocities in the body frame } \\ N_{x b} N_{y b} N_{z b} & \text { Wind velocities in the inertial } \\ p_{b} q_{b} r_{b} & \text { frame } \\ \text { Suffix 'm' } & \text { Position vector } \\ p q r & \text { Accelerometer bias } \\ N_{x} N_{y} N_{z} & \text { Gyro bias } \\ & \text { Indicates measurement } \\ & \text { Air Data System } \\ & \text { Rates } \\ & \text { Accelerations } \\ & \end{array}$

\section{References}

[1] Jategaonkar R V 2006 Data compatibility check. In: Flight Vehicle System Identification A Time Domain Methodology. Chapter 10, Volume 216, Reston, Virginia, AIAA, pp. 335-350

[2] Morelli E A 2002 System Identification Programs for Aircraft (SIDPAC). AIAA, Reston

[3] Morelli E A 2003 Low-order equivalent system identification for the Tu-144LL supersonic transport aircraft. J. Guid. Control Dyn. 26: 354-362

[4] Morelli E A and Klein V 2005 Application of system identification to aircraft at NASA Langley Research Center. $J$. Aircraft 2: 12-25

[5] Mulder J A, Chu Q P, Sridhar J K, Breeman J H and Laban M 1999 Non-linear aircraft flight path reconstruction review and new advances. Prog. Aerosp. Sci. 35: 673-726

[6] Kamali C, Jain S, Sarf A and Goyal A 2016 Calibration of static pressure sensors using Extended Kalman Filter at high angles of attack and transonic mach numbers. IEEE Xplore. http://doi.org/10.1109/INDIANCC.2016.7441170 
[7] Jain S, Kamali C, Yarlagadda Y, Saraf A and Goyal A 2017 Calibration and accuracy determination of airdata system for a modern fighter. AIAA SciTech Forum, (AIAA 2017-1868). https://doi.org/10.2514/6.2017-1868

[8] Kamali C, Jain S and Saraf A 2016 Novel method for static pressure calibration using Unscented Kalman Filter. IFACPapersOnLine 49: 646-651. https://doi.org/10.1016/j.ifacol. 2016.03.129

[9] Kamali C, Jain S, Yarlagadda Y and Saraf A 2017 Airdata calibration using error state filter. In: AIAA Atmospheric Flight Mechanics Conference, AIAA AVIATION Forum, (AIAA 2017-3895). https://doi.org/10.2514/6.2017-3895

[10] Evans R J, Goodwin G C, Feik R A, Martin C and LozanoLeal R 1985 Aircraft flight data compatibility checking using maximum likelihood and Extended Kalman Filter estimation. IFAC 18: 487-492
[11] Girija G and Raol J R 1993 PC based flight path reconstruction using UD factorization filtering algorithm. Def. Sci. J. 43: 429-447

[12] Parameswaran V and Jategaonkar R V 2004 Calibration of 5 hole probe for flow angles from advanced technologies testing aircraft system flight data. Def. Sci. J. 54: 111123

[13] Nusrath K, Sarmah A and Singh J 2014 Flight path reconstruction and wind estimation using flight test data from Crash Data Recorder (CDR). SAE Technical Paper 2014-012168, 2014. https://doi.org/10.4271/2014-01-2168

[14] Majeed M and Kar I N 2011 Multi sensor data fusion based approach for the calibration of airdata systems. Aeronaut. J. 115: 113-122

[15] Etkin B 1972 Dynamics of atmospheric fight. Wiley, New York 